\title{
Splenium of the Corpus Callosum
}

National Cancer Institute

\section{Source}

National Cancer Institute. Splenium of the Corpus Callosum. NCI Thesaurus. Code C33610.

The posterior bulbous part of the corpus callosum situated, which is located anterior to the occipital lobe, and which overhangs the thalamic pulvinars, the pineal gland and tectum of the midbrain; Its fibers connect occipital and parietal cortices, as well as inferior and medial temporal regions. 